\title{
Perú 2011: Continuidades y CAMbios EN la POlítica SIN PARTIDOS
}

Peru 2011: Continuities and changes in politics without parties

\section{EDUARDO DARGENT}

Pontificia Universidad Católica del Perú

\section{PAULA MUÑOZ}

Universidad de Texas en Austin

\begin{abstract}
RESUMEN
La elección de Ollanta Humala en mayo del 2011 hizo pensar que este año podrían darse cambios sustanciales en la política peruana. Si bien en el 2011 el Perú sí experimentó cambios políticos que dejan algunas preguntas abiertas sobre el futuro, la imagen que presentamos en este reporte es fundamentalmente una de continuidad, tanto en la forma de hacer política como en los factores que afectan la misma. En este reporte nos centramos en la política peruana en el 2011, aunque en algunos casos que lo ameriten revisamos eventos del 2010. Resaltamos aquellos aspectos que creemos hacen interesante al caso peruano desde una perspectiva comparada.
\end{abstract}

Palabras clave: Perú, democracia, conflictos sociales, elecciones, partidos políticos.

\begin{abstract}
The election of Ollanta Humala in May 2011 made some observers speculate that this year could signal substantial changes in Peruvian politics. Even if Peru did witness some political changes that did leave questions open about possible future change, the image presented in this report is fundamentally one of continuity, both in the way in which politics is conducted as well as in the factors that affect politics. While this report focuses on Peruvian politics during 2011, it reviews some cases from 2010 that deserve attention as well. Those aspects that make the Peruvian case interesting from a comparative perspective are highlighted throughout.
\end{abstract}

Key words: Peru, democracy, social conflicts, elections, political parties. 


\section{INTRODUCCIÓN}

La elección del candidato reformista Ollanta Humala en mayo del 2011 hizo pensar que este año podrían darse cambios sustanciales en la política peruana. Si bien este año el Perú sí experimentó cambios políticos que dejan algunas preguntas abiertas sobre el futuro, la imagen que presentamos en este reporte es fundamentalmente una de continuidad. El nivel de institucionalización del sistema político sigue siendo muy bajo, con instituciones desprestigiadas y partidos débiles. Asimismo, ayudado por otro año más con precios internacionales altos de los minerales, Perú logró limitar el impacto de la crisis económica del 2009 y sigue liderando el crecimiento económico en Sudamérica. Los grupos empresariales, particularmente aquellos que acumularon poder tras las reformas de mercado de los noventa como exportadores, financieros y mineros, siguen siendo actores políticos de mucho peso. El Estado confronta aún serios problemas para controlar actividades ilegales en el territorio, como los rezagos de Sendero Luminoso, el narcotráfico, la minería informal y el contrabando.

Asimismo, dos factores que fueron identificados como los pilares de un precario equilibrio de gobernabilidad en un anuario anterior (Meléndez y León, 2010), la debilidad de la oposición institucional y la persistente pero fragmentada protesta social vinculada en su mayoría a temas socioambientales, se mantienen. Finalmente, como veremos, es probable que el "reformista" Humala mantenga al Poder Ejecutivo en una posición similar a la de los anteriores presidentes postransición, Alejandro Toledo y Alan García.

Parte de estas continuidades se explican por un crecimiento económico alto con efectos desiguales en el territorio. La expansión económica iniciada en los 2000 bajo el modelo neoliberal de desarrollo ha acentuado y politizado las desigualdades regionales en el Perú (Muñoz, 2009). Mientras que, particularmente, Lima y la costa peruana logran engancharse al modelo agroexportador, otras zonas del país como la sierra sur y la selva no obtienen similares beneficios del crecimiento. Por ello, la elasticidad del crecimiento económico a la pobreza es heterogénea regionalmente (Banco Mundial, 2008). Los efectos políticos de este fenómeno fueron muy claros en las elecciones de 2006 cuando el voto siguió patrones regionales que se repitieron, con matices, en la elección del 2011. La dinámica "neodualista" identificada por Tanaka y Vera en un reporte anterior (2008) y patrones de conducta electoral determinados por la relación de los votantes con el despliegue tanto de las atribuciones estatales como del mercado sobre el territorio identificados por Alberto Vergara (2007), marcan la política electoral peruana.

Dentro de esta imagen de continuidad, también hay algunos cambios cuyas consecuencias son todavía difíciles de evaluar. La popularidad del presidente Humala se mantiene alta seis meses después de iniciar su gobierno (Gráfico 1). Dicha aprobación es bastante uniforme a través de regiones y clases sociales. Si bien sus críticos señalan que con su movimiento hacia la derecha después de la elección Humala abandonó a parte de su base electoral, las encuestas muestran que hasta el momento el gobierno mantiene el apoyo de sectores pobres que lo apoyaron durante la elección. ${ }^{1}$ Es decir, parte de su base

1 Hay que tener en cuenta, sin embargo, el sesgo urbano de las encuestas de aprobación presidencial. 
electoral no vería ese movimiento como una "traición" electoral, quizás por el inicio de una serie de programas sociales dirigidos a los grupos de extrema pobreza.

Gráfico 1: Aprobación presidente Humala

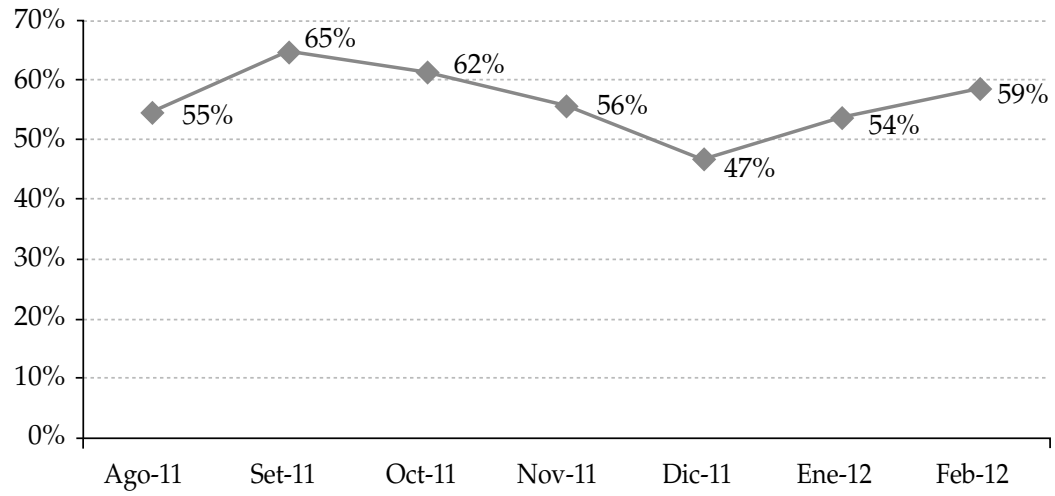

Fuente: IPSOS-APOYO.

La pregunta abierta es si al final de su mandato Humala se parecerá a Alan García y Alejandro Toledo, Presidentes que terminaron con una popularidad media/baja, sostenida por su apoyo en zonas urbanas y clases medias y altas, o si logrará consolidar su base actual de apoyo a través de regiones y clases sociales antes críticas del gobierno, ganando así mayor legitimidad que sus predecesores. En este reporte nos centramos en la política peruana del 2011, aunque en algunos casos que lo ameriten revisamos eventos del 2010. Resaltamos aquellos aspectos que creemos hacen interesante al caso peruano desde una perspectiva comparada.

\section{TEMAS SALIENTES EN LA REALIDAD NACIONAL Y SU IMPACTO POLÍTICO}

\section{i. Coyuntura política}

El último año del gobierno aprista (julio 2010-julio 2011) estuvo marcado por los procesos electorales subnacionales y nacionales, discutidos en la sección 4 en detalle. Como rasgos generales de estas elecciones podemos adelantar que ellas confirmaron la debilidad de los partidos peruanos, una situación que no han logrado revertir desde su colapso en los tempranos noventa (Levitsky y Cameron, 2003; Tanaka, 2005).

La segunda mitad del año el debate político se centró en las expectativas sobre la posición en el espectro ideológico del nuevo gobierno del presidente Humala. Algunas medidas iniciales mostraron a Humala buscando aproximarse a su base electoral de primera vuelta, aunque también dejando en claro que garantizaba la continuidad y estabilidad económica. Estas acciones lo colocaron en una cómoda posición de centro, logrando apoyo considerable en todos los niveles sociales. Parte de esta imagen se ve 
quebrada con la actitud del gobierno para resolver un conflicto minero en la región de Cajamarca (sección 1.ii), interpretado por algunos de sus aliados de izquierda como un rompimiento con un sector de su base electoral de primera vuelta.

Este movimiento hacia la derecha hasta el momento no ha tenido efectos negativos sobre la popularidad de Humala. El gobierno alcanza niveles de aprobación muy superiores a los de Toledo en el mismo período, y mejores que los de García, aunque la aprobación de este último recién empieza a caer sostenidamente ocho meses después de iniciar su gobierno. En la sección final especulamos sobre cómo nuevos programas sociales y otras medidas le permitirían mantener su popularidad entre los sectores bajos.

\section{ii. Coyuntura social}

Como años anteriores, el 2011 tuvo varias protestas sociales a nivel local, la mayoría relacionadas de una u otra manera con actividades extractivas. Durante el 2010 el número de conflictos sociales activos bajó en relación al pico que se observara durante el 2009 (Gráfico 2) y se mantendrá más o menos estable alrededor de una media de 150 conflictos activos durante el 2011. Más que el reflejo de una nueva estrategia en el manejo de los conflictos por parte del gobierno, este freno podría explicarse por el inicio de la competencia electoral y la recuperación del crecimiento económico (ver Gráfico 2).

Gráfico 2: Número de conflictos sociales activos por mes (abril 2008-diciembre 2011)

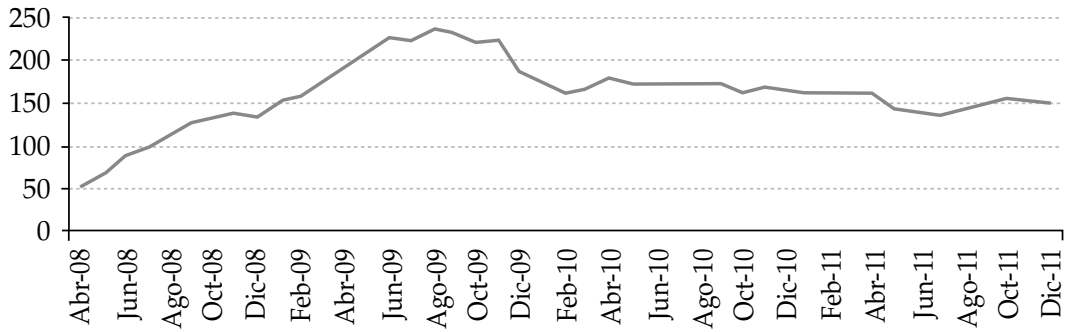

Fuente: Defensoría del Pueblo.

La protesta de la población local suele asociarse a los impactos socioambientales del proyecto en cuestión y/o a la demanda de mayores recursos/beneficios para la población local. Los números de concesiones mineras aprobadas por el Ministerio de Energía y Minas durante la década muestran que ha existido una intensa política de promoción de la actividad minera desde el gobierno central (Cooperacción, 2010: 5). Según sus críticos, el Ministerio de Energía y Minas favorece la promoción de la inversión, sin tener el cuidado suficiente al evaluar los costos ambientales y sociales de los proyectos. Esta situación da lugar a casos en que millonarios proyectos de inversión aprobados por el Ejecutivo finalmente son paralizados por protestas locales. El gobierno central no es capaz de controlar la dinámica local. 
Entre marzo y abril el conflicto por el inicio de actividades de un proyecto cuprífero de la empresa Southern Copper en Arequipa (Proyecto Tía María) dio lugar a enfrentamientos entre la población y el gobierno nacional. Finalmente el proyecto fue cancelado por el gobierno aduciendo problemas con el Estudio de Impacto Ambiental (EIA) presentado por la empresa, la cual se ha comprometido a realizar un nuevo estudio tomando en cuenta estas observaciones. Asimismo, en junio y julio una serie de protestas en Puno dejaron ver los complejos entramados del tema minero en dicho departamento y la ineficiencia del Estado para responder ante ellos: chocaron intereses de la minería formal, minería informal, los productores agropecuarios, el contrabando, empresarios locales vinculados al turismo, haciendo muy difícil acuerdos satisfactorios para las partes en disputa. Luego, recién inaugurado el gobierno de Ollanta Humala, se realizaron operativos militares y policiales para destruir una serie de dragas artesanales utilizadas por mineros informales para extraer oro del río Madre de Dios.

Durante la campaña electoral Ollanta Humala prometió atender las demandas de poblaciones afectadas por empresas extractivas, siendo el candidato favorecido por sectores críticos de la minería. En referencia al tema minero en Cajamarca, el candidato Humala había señalado que si había que escoger entre el oro y el agua, su gobierno privilegiaría el agua. Paradójicamente, Humala tuvo su primera prueba de fuego como gobernante al enfrentar las protestas por la aprobación de un proyecto aurífero de la empresa Yanacocha (Minas Conga) en dicha región. El proyecto original, aprobado durante el gobierno de Alan García y de acuerdo a sus críticos sin contar con un EIA adecuado, afecta cuatro lagunas.

Desde octubre crecieron las protestas contra este proyecto, lideradas por el presidente regional de Cajamarca Gregorio Santos y otros líderes locales. El presidente del Consejo de Ministros de Ollanta Humala, el empresario Salomón Lerner Ghitis, intentó dialogar con los dirigentes cajamarquinos, pero fracasó en lograr un acuerdo. El gobierno declaró el Estado de Emergencia en cuatro provincias. Previamente Yanacocha había suspendido los trabajos en el proyecto a pedido del gobierno. Estas protestas y el fracaso de las negociaciones dieron lugar a un cambio de gabinete apenas cuatro meses después de iniciado el gobierno. Al parecer la decisión de adoptar el Estado de Emergencia se tomó contra la opinión del premier Lerner, motivando su renuncia. Fue reemplazado por el entonces ministro del Interior, Óscar Valdez, militar en retiro y percibido como un "halcón" durante las negociaciones. El gobierno ha determinado que un grupo de peritos extranjeros evalúe el EIA presentado por la empresa y aprobado por el Ministerio de Energía y Minas, aunque los dirigentes locales dudan de la imparcialidad de dichos peritos.

Si bien estos conflictos fueron bastante serios, creemos que siguen el patrón de años anteriores: casos de protesta local, con impacto reducido en otras regiones, y sin desestabilizar al gobierno. La protesta no escala. Lo particular de la reciente protesta en Cajamarca, sin embargo, es que por primera vez un presidente regional toma un papel tan claro de liderazgo en el conflicto. El año terminó con la convocatoria y preparación de la Marcha por el Agua, un esfuerzo de diversos líderes locales por darle al tema de la minería y el uso del agua carácter nacional. Por la fragmentación antes señalada, estos esfuerzos son difíciles de concretar. 


\section{iii. Coyuntura económica}

El año 2010 observó la recuperación del crecimiento económico que había caído el 2009 como resultado de la crisis económica internacional. A pesar de las apocalípticas predicciones sobre el impacto de un triunfo de Ollanta Humala en la economía, el 2011 fue un año relativamente bueno en términos económicos (Gráfico 3). La pobreza se sigue reduciendo, aunque con las diferencias regionales antes resaltadas.

Desde que Humala creció en las encuestas a inicios de año, se inició un período de dudas y rumores sobre el futuro de la economía. El gobierno elegido tomó medidas rápidas para ganar la confianza entre los inversionistas y empresarios. Estas medidas consistieron en mostrar su voluntad de respetar la tecnocracia instalada en los centros de decisión económica del Estado, como el Ministerio de Economía y Finanzas y el Banco Central. Humala parece haber dejado de lado sus duras críticas al modelo económico, para hacerlo uno de los activos de su gobierno. En sus apariciones públicas Humala ha resaltado la necesidad de un alto crecimiento económico como requisito para combatir la pobreza y la desigualdad, lo que ha llamado "crecimiento con inclusión".

Gráfico 3: Crecimiento económico anual 2001-2011

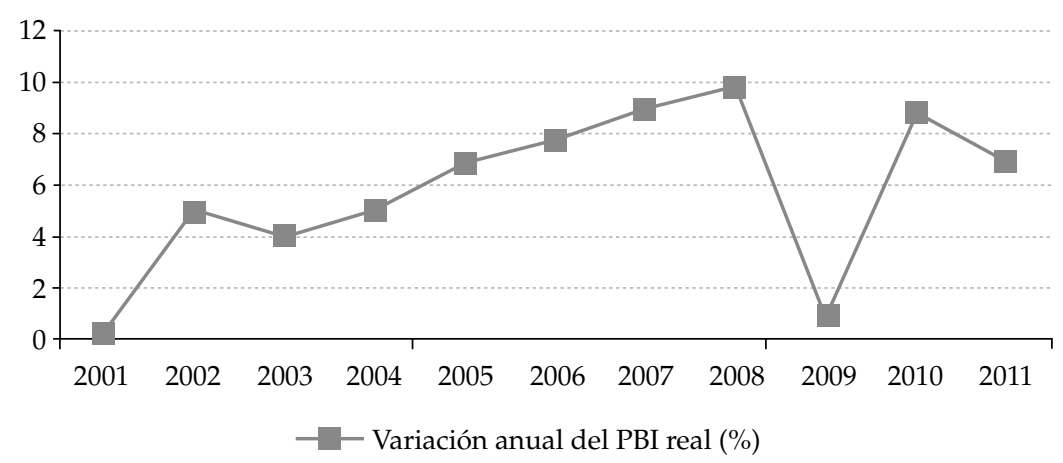

Fuente: BCR.

Gráfico 4: Incidencias de la pobreza total y extrema 2004-2010 (porcentaje)

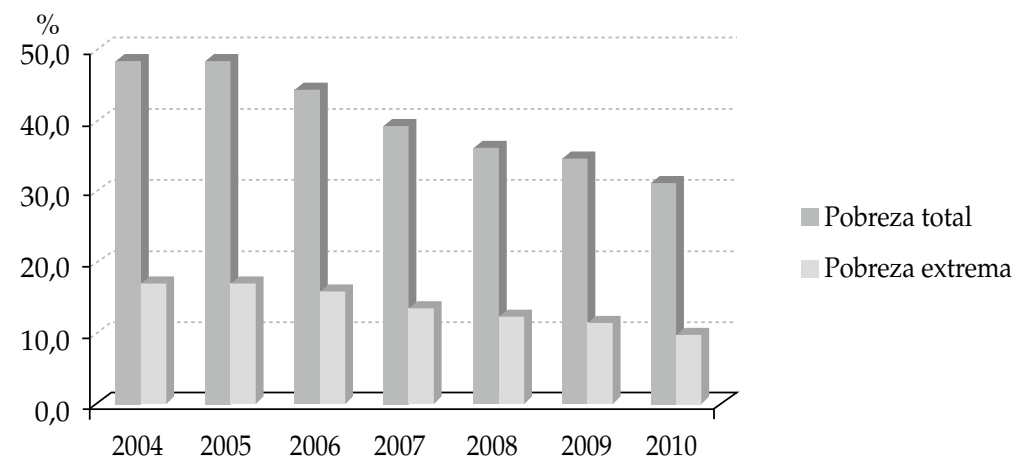

Fuente: ENAHO-INEI. 


\section{iv. Coyuntura subregional e internacional}

Aunque había expectativas sobre un posible acercamiento de un gobierno del presidente Humala al grupo de países conformado por Venezuela y Bolivia, el gobierno fue rápido en asegurar que su política exterior no diferenciaría entre ejes, "amigos" o "enemigos". Giras realizadas a diferentes países después de la elección, y otros viajes ya durante su mandato, muestran esta vocación por mantener buenas relaciones multilaterales.

Desde la campaña presidencial ha sido clara la creciente influencia de Brasil en el Perú. Asesores electorales brasileños, muy cercanos al Partido de los Trabajadores, fueron clave en el diseño de una estrategia electoral exitosa y se mencionan empresas brasileñas como financistas del Partido Nacionalista (León, 2011). El tema es relevante pues un memorando del año 2009 firmado por los Presidentes de Perú y Brasil señala el compromiso de este país de invertir en seis proyectos hidroeléctricos en territorio peruano. Esta energía será vendida al mercado brasileño. El más conocido de estos megaproyectos es el de la represa de Inambari, impulsado por la empresa brasileña OAS y la peruana EGASUR. La concesión de este proyecto, no obstante, no fue renovada el 2011 debido a que la empresa no habría cumplido con informar a la población local sobre el impacto socioambiental del mismo. Esta decisión estuvo precedida por una intensa protesta de organizaciones sociales y autoridades locales en contra el proyecto.

También relevante a nivel de coyuntura internacional es el litigio iniciado por el Perú en el 2008 contra Chile por la delimitación de la frontera marítima ante la Corte Internacional de La Haya. El proceso agotó su fase escrita este año. Durante el año hubo algunas declaraciones altisonantes por parte de autoridades de lado y lado, pero el tono hasta ahora ha sido bastante respetuoso.

\section{CAMBIOS INSTITUCIONALES (Y CONSTITUCIONALES) Y CAMBIOS SUBSTANCIALES EN POLÍTICAS PÚBLICAS}

Los cambios institucionales más notorios previos al 2011 fueron las reformas para la elección de gobiernos regionales, la creación de un nuevo distrito electoral y una reforma constitucional que incrementa en diez congresistas la representación nacional. Con el objetivo de evitar que la fragmentación existente en el país diera lugar a gobiernos regionales elegidos por una minoría se adoptó en el 2009 una segunda vuelta electoral en aquellas regiones en las que un candidato no alcanzara el 30\% de los votos (Ley $\mathrm{N}^{\mathrm{o}} 29.470$ ). Asimismo, esta ley adopta distritos provinciales uninominales para elegir a los representantes del Consejo Regional con el objetivo de forzar a los candidatos regionales a construir redes y hacer campañas en las provincias. De acuerdo a la legislación anterior se otorgaba al movimiento ganador la mayoría del Consejo a pesar de no obtenerla en las urnas, y el resto de consejeros se repartían proporcionalmente.

Asimismo, en el 2010 se reformó la Constitución (artículo 90) para incrementar de 120 a 130 el número de congresistas en el Congreso (unicameral). Este aumento de congresistas se realizó en parte como resultado de un debate sobre la necesidad de crear un nuevo 
distrito electoral que separe la representación de las provincias del departamento de Lima de la provincia de Lima (donde se ubica la capital). El Congreso reformó la Ley Orgánica de Elecciones, Ley $\mathrm{N}^{\circ}$ 26859, creando el distrito electoral Lima Provincias. Ello constituye un sistema electoral con 26 distritos electorales, conformados por los 24 departamentos, la Provincia Constitucional del Callao y ahora el Distrito Electoral de Lima Provincias (ver Cuadro 1).

Conforme a la ley electoral, el Jurado Nacional de Elecciones (JNE) estuvo a cargo de asignar los diez nuevos representantes. Los 10 escaños se asignaron de la siguiente manera: cuatro para la nueva circunscripción, y un escaño adicional para Arequipa,

Cuadro 1: Magnitud de Distritos Electorales para elección del Legislativo

\begin{tabular}{|c|c|c|}
\hline Distrito & Escaños & Electores hábiles \\
\hline Amazonas & 2 & 231.137 \\
\hline Ancash & 5 & 737.514 \\
\hline Apurimac & 2 & 244.008 \\
\hline Arequipa & 6 & 892.068 \\
\hline Ayacucho & 3 & 369.929 \\
\hline Cajamarca & 6 & 891.597 \\
\hline Callao & 4 & 649.896 \\
\hline Cusco & 5 & 778.328 \\
\hline Huancavelica & 2 & 252.618 \\
\hline Huanuco & 3 & 447.386 \\
\hline Ica & 4 & 517.529 \\
\hline Junin & 5 & 786.304 \\
\hline La Libertad & 7 & 1.115 .648 \\
\hline Lambayeque & 5 & 784.633 \\
\hline Lima-Extranjero & 36 & 6.745 .985 \\
\hline Lima-Provincias & 4 & 616.283 \\
\hline Loreto & 4 & 544.358 \\
\hline Madre de Dios & 1 & 71.279 \\
\hline Moquegua & 2 & 121.977 \\
\hline Pasco & 2 & 167.179 \\
\hline Piura & 7 & 1.106 .918 \\
\hline Puno & 5 & 784.979 \\
\hline San Martin & 4 & 467.689 \\
\hline Tacna & 2 & 216.784 \\
\hline Tumbes & 2 & 138.509 \\
\hline Ucayali & 2 & 269.380 \\
\hline Total & 130 & 19.949 .915 \\
\hline
\end{tabular}

Fuente: ONPE. 
Cajamarca, Lima, Loreto, Piura y San Martín. Lo interesante del caso en perspectiva comparada es la poca atención y debate público sobre este proceso. El debate se limitó a los esfuerzos de la bancada del Partido Nacionalista para que la creación del nuevo distrito electoral se realizara sin incrementar el número total de congresistas. Al parecer los nacionalistas preferían que la creación del nuevo distrito electoral redujera el número de representantes elegidos hasta ese momento por Lima (35) dada la debilidad electoral de Humala en dicha circunscripción. El resto de grupos políticos prefirió ampliar el número de congresistas en vez de cambiar la distribución actual. Además de Lima-Provincias, los seis congresistas adicionales aumentaron la representación de sectores del país que han tenido mayor crecimiento demográfico en estos años. Si bien es necesario investigar más el tema, la decisión del JNE parece ser técnica y siguiendo criterios demográficos.

Otras reformas importantes implementadas este año tienen que ver con las promesas de campaña de Humala. El nuevo Congreso aprobó por unanimidad la Ley de Consulta Previa, Ley $\mathrm{N}^{\circ}$ 29785. Dicha norma manda al gobierno a consultar a las comunidades indígenas antes de aprobar actividades extractivas en su territorio, conforme a lo señalado en el Convenio 169 de la Organización Internacional del Trabajo. La norma todavía debe reglamentarse y el debate actual se centra, básicamente, en cuáles serán las consecuencias legales de dicha consulta comunal.

Asimismo, conforme a lo ofrecido en campaña, el gobierno también determinó un aumento considerable en la contribución de las empresas mineras al fisco al aprobar la Ley de Sobreganancias mineras, Ley N ${ }^{\circ}$ 29788. El gobierno calcula una recaudación de 3,000 millones de soles anuales con este gravamen, seis veces superior al llamado óbolo minero aprobado durante el gobierno de García. La nueva regalía se calcula en relación con las utilidades de la empresa, evitando así afectar su competitividad, e incluye a empresas mineras que gozaban de convenios de estabilidad.

Finalmente, se creó el Ministerio de Desarrollo e Inclusión Social que agrupa a una serie de programas sociales antes dispersos en el Estado. Este nuevo súper ministerio social ha sido encargado a una economista independiente y viene realizando una importante gestión de racionalización y expansión de estos programas, así como el lanzamiento de otros nuevos prometidos durante la campaña (discutimos más sobre ellos en la última sección).

\section{RESULTADOS DE ELECCIONES NACIONALES Y SUBNACIONALES}

El 2010 fue el primer año desde 1989 que las elecciones subnacionales preceden a las generales por unos meses. Los peruanos eligieron a sus autoridades municipales y regionales el 3 de octubre del 2010. El 5 diciembre 10 departamentos votaron también una segunda vuelta para elegir a sus presidentes regionales. Las elecciones presidenciales y congresales tuvieron lugar el 10 de abril de 2011 y la segunda vuelta presidencial se realizó el 5 de junio. Es decir, Perú vivió, literalmente, en campaña electoral por casi un año.

Estos procesos electorales estuvieron cargados de incertidumbre dada la volatilidad de las preferencias electorales. No obstante, una evaluación de los mismos permite 
identificar algunas tendencias claras en medio de la improvisación e inestabilidad de las opciones políticas que caracterizan a la política peruana.

En primer lugar, las elecciones del 2010 y 2011 muestran, una vez más, la debilidad de los partidos políticos peruanos; debilidad que subsiste a pesar de las reformas implementadas desde inicios de los 2000 con la finalidad de fortalecerlos y favorecer su crecimiento. Por un lado, las elecciones subnacionales confirmaron la incapacidad de los partidos políticos nacionales para ganar elecciones a nivel local (Tanaka y Guibert, 2011; Remy, 2011). Tal como sucedió en las elecciones subnacionales del 2006, los ganadores de los comicios del 2010 fueron los movimientos regionales (Cuadros 2 y 3). De hecho, el éxito relativo de estos partidos regionales es un fenómeno extraño a nivel comparado, sobre todo por darse en un país sin movimientos regionalistas/separatistas y sin identidades étnicas politizadas (De Gramont, 2010: 25).

Por otro, ambos procesos electorales mostraron en particular el retroceso de partidos nacionales tradicionales, como el APRA y el PPC, cuyos mejores resultados al inicio de la década dieron esperanzas de un supuesto resurgimiento parcial del antiguo sistema de partidos (Kenney, 2003). Lourdes Flores, lideresa del PPC que quedó tercera en las elecciones presidenciales del 2001 y 2006 como candidata de la alianza de derecha Unidad Nacional, pierde una vez más una elección. Esta vez fue la alcaldía de Lima Metropolitana, su mejor cancha electoral, y contra una candidata de izquierda bastante inexperta. Si bien la izquierdista Susana Villarán venía subiendo lentamente en las preferencias, Villarán creció en las encuestas cuando el hasta entonces principal competidor de Flores, Álex

Cuadro 2: Número de gobiernos regionales ganados según tipo de agrupación política

\begin{tabular}{lccc}
\hline Tipo de agrupación política & 2002 & 2006 & 2010 \\
\hline Partidos & 18 & 8 & 9 \\
Movimientos regionales & 7 & 17 & 16 \\
\hline Total & 25 & 25 & 25 \\
\hline
\end{tabular}

Tomado de: Tanaka y Guibert, 2011. Fuente: ONPE.

Cuadro 3: Número de municipalidades provinciales ganadas según tipo de agrupación política

\begin{tabular}{lrrr}
\hline Tipo de agrupación política & 2002 & 2006 & 2010 \\
\hline Partidos & 110 & 109 & 72 \\
Movimientos regionales & 30 & 69 & 117 \\
Organizaciones locales & 54 & 17 & 6 \\
\hline Total & 194 & 195 & 195 \\
\hline
\end{tabular}

Tomado de: Tanaka y Guibert, 2011. Fuente: ONPE. 
Kouri, presidente regional del Callao, fue retirado por el Jurado Nacional de Elecciones de la campaña por no cumplir con un requisito de la ley electoral. Villarán se impuso a Flores por menos de $1 \% .^{2}$

La elección nacional mostró que el APRA tiene poca fuerza electoral cuando Alan García no es su candidato. Dicho partido no logró presentar un candidato presidencial en el 2011 a pesar de las buenas cifras económicas del gobierno. ${ }^{3}$ Con cuatro congresistas y gobernando apenas en una región, el futuro del APRA aparece más atado que nunca a la suerte de Alan García.

El partido que sí se fortaleció en este período fue el Fujimorista. Bajo el liderazgo de Keiko Fujimori el fujimorismo volvió a ser protagonista en una elección nacional. No es exagerado señalar que se ha convertido en la fuerza política más organizada del país, aunque todavía lejos de ser un partido sólido (Navarro, 2011; Urrutia, 2011).

En segundo lugar, estos procesos electorales muestran de manera más general los problemas que confrontan los políticos peruanos para coordinar sus acciones a través de distritos electorales y de esa forma articular el sistema político. Como sostienen Muñoz y García (2011), el Perú es un caso exagerado de desarticulación política a través de sus cuatro niveles de gobierno (nacional, regional, provincial y distrital). El problema no es solamente que los partidos nacionales son incapaces de competir exitosamente a nivel subnacional y así garantizar su presencia en el territorio (Vergara, 2012). Con algunas contadas excepciones, los movimientos regionales tampoco son partidos organizados capaces de ganar elecciones municipales al interior de su región y, a su vez, las agrupaciones provinciales tampoco en los distritos (Muñoz y García, 2011). Desde esta perspectiva de ausencia de articulación a través de distritos electorales, el problema de la política peruana va más allá de la mera fragmentación política. Dicho sea de paso, la fragmentación tampoco ha variado substancialmente con respecto a los anteriores procesos electorales. ${ }^{4}$

Relacionado con los dos puntos anteriores, estos procesos electorales muestran también cómo precisamente la debilidad organizativa en la política da un mayor margen para que el azar y la contingencia impacten los resultados de la competencia política. Sin contrapesos organizacionales, las decisiones (aciertos y desaciertos) de políticos en posiciones de liderazgo adquieren mayor relevancia. Al mismo tiempo, sin organizaciones partidarias consolidadas y duraderas que constriñan a sus integrantes a la lógica e intereses del grupo, los socialicen y faciliten el aprendizaje colectivo, es muy fácil que improvisadas alianzas electorales cometan errores garrafales a lo largo de la campaña. ${ }^{5}$

2 Luego de su victoria, sin embargo, la popularidad de la Alcaldesa declinó drásticamente a través del año. Su popularidad cayó por debajo del $20 \%$ a fines de año y en la actualidad ciertos grupos han iniciado la recolección de firmas para solicitar su revocatoria.

3 Para un balance del APRA ver Cyr, 2011 y Vergara, 2011.

4 El número de partidos efectivos promedio de las elecciones regionales del 2002, 2006 y 2010 son 5,63, 5,27 y 5,44 respectivamente (Muñoz y García, 2011: 12). El número de partidos efectivos en las elecciones presidenciales de 2001, 2006 y 2011 son, según nuestros cálculos, 3,72, 4,54 y 4,45 respectivamente.

5 Ver por ejemplo el análisis que Meléndez, 2011 y Vera, 2011 realizan de las campañas de Castañeda y Toledo, respectivamente. 
En suma, la debilidad partidaria hace que los eventos y estrategias de campaña puedan pesar bastante en la definición de los resultados (Levitsky, 2011). La elección presidencial del 2011 dio cuenta de esto. De hecho, tal vez con la excepción parcial del fujimorismo, el resto de los candidatos en contienda lideraban partidos personalistas.

Dada la debilidad generalizada de las identidades partidarias, la campaña mostró cambios drásticos en la intención de voto. Al inicio el ex presidente Alejandro Toledo trepó en las preferencias electorales hasta febrero del 2011, apareciendo como ganador o cuando menos seguro participante en segunda vuelta con intención de voto superior al 35\%. Bastante detrás de él estaba Keiko Fujimori, con alrededor de 22\% a lo largo de toda la campaña. Luis Castañeda se ubicó en tercer lugar, aunque cayendo lentamente mes a mes. Y Ollanta Humala no lograba despegar hasta entrado el 2011. En marzo del 2011 todo cambió, con Humala creciendo aceleradamente. La subida de Humala en la intención de voto fue acompañada por una aguda e incierta pugna por el segundo lugar. Semana a semana Toledo empezó a caer en las encuestas mientras que la candidatura del ex ministro de Economía, Pedro Pablo Kuczynski, empezaría a crecer a partir de marzo. Según Ipsos Apoyo, a fines de marzo y a dos semanas de la elección, apenas siete puntos separaban al primero (Humala con $21 \%$ ) del quinto (Castañeda con 14\%). Finalmente Humala obtuvo la primera mayoría con 32\% de los votos. Segunda quedó Fujimori (24\%) y tercero Kuczynski con 19\% (Cuadro 4).

En buena medida, los resultados de la primera vuelta de la elección presidencial tuvieron mucho que ver con los problemas de coordinación de vehículos personalistas para la definición de candidaturas. Pero también en los problemas de coordinación que los electores del campo de centro-derecha tuvieron para apoyar consistentemente una sola candidatura.

Este resultado tan fragmentado llevó a una segunda vuelta muy polarizada con dos candidatos que dejaban dudas sobre sus calidades democráticas, y con dos propuestas distintas sobre la continuidad del modelo de mercado. Humala hizo de la democracia una de sus banderas, cuidándose además de lanzar señales de moderación económica (León, 2011). Particularmente al pasar a la segunda vuelta presentó programas y documentos que constituían un compromiso público de moderación (Hoja de Ruta y Compromiso por la Democracia). El papel de líderes de opinión democrática, como Mario y Álvaro Vargas Llosa y Alejandro Toledo resultó clave para darle credibilidad a la moderación de Humala. Fujimori, por su parte, centró su campaña de segunda vuelta en la continuidad económica e hizo pocos gestos de moderación democrática. Su candidatura contó con el respaldo de la clase alta y el empresariado. Asimismo, los medios de comunicación nacionales perdieron rigurosidad periodística en su cobertura de la segunda vuelta y apostaron, mayoritaria y abiertamente, por apuntalar la candidatura de Fujimori y perjudicar la de Humala (Llorens, 2011).

Finalmente, en esta segunda vuelta la clase media urbana se divide y termina inclinando ligeramente la balanza a favor de Humala tras mucha incertidumbre. El movimiento al centro de Humala dio resultado, ganando la elección por un margen de 2,8\% de los votos (Cuadro 5). Su estrategia fue exitosa pues logró que sus votantes en la segunda vuelta lo 
Cuadro 4: Resultados de las Elecciones Presidenciales 2011 - Primera Vuelta

\begin{tabular}{llrc}
\hline Candidato & \multicolumn{1}{c}{ Partido } & Votos & \% votos válidos \\
\hline Ollanta Humala & Gana Perú & 4.643 .064 & 31,72 \\
Keiko Fujimori & Fuerza 2011 & 3.449 .595 & 23,57 \\
Pedro Pablo Kuczynski & Alianza por el Gran Cambio & 2.711 .450 & 18,52 \\
Alejandro Toledo & Perú Posible & 2.289 .561 & 15,64 \\
Luis Castañeda & Alianza Solidaridad Nacional & 1.440 .143 & 9,84 \\
José Antonio Nique & Fonavistas del Perú & 37.011 & 0,25 \\
Ricardo Noriega & Despertar Nacional & 21.574 & 0,15 \\
Rafael Belaúnde & Adelante & 17.301 & 0,12 \\
Juliana Reymer & Fuerza Nacional & 16.831 & 0,12 \\
Humberto Pinazo & Justicia, Tecnología, Ecología & 11.275 & 0,08 \\
\hline Total votos & & 14.637 .805 & 100,00 \\
Votos válidos & & 14.637 .805 & 87,70 \\
Votos blancos & & 1.477 .696 & 8,80 \\
Votos nulos & & 584.233 & 3,50 \\
Total votos emitidos & & 16.699 .734 & 100,00 \\
Participación & & 16.699 .734 & 83,70 \\
\hline Total electores hábiles & & 19.949 .915 & 100,00 \\
\hline
\end{tabular}

Fuente: ONPE.

Cuadro 5: Resultados de las Elecciones Presidenciales 2011 - Segunda Vuelta

\begin{tabular}{lccc}
\hline Candidato & Partido & Votos & \% votos válidos \\
\hline Ollanta Humala & Gana Perú & 7.937 .704 & 51,4 \\
Keiko Fujimori & Fuerza 2011 & 7.490 .647 & 48,6 \\
\hline Total votos & & 15.428 .351 & 100,0 \\
Votos válidos & 15.428 .351 & 93,7 \\
Votos Blancos & 116.335 & 0,7 \\
Votos Nulos & 921.711 & 5,6 \\
Total votos emitidos & 16.466 .397 & 100,0 \\
Participación & 16.466 .397 & 82,5 \\
Ausentes & 3.483 .518 & 17,5 \\
\hline Total electores hábiles & 19.949 .915 & 100,0 \\
\hline
\end{tabular}

Fuente: ONPE. 
percibieran como ideológicamente más moderado (Muñoz, 2011). En última instancia, gran parte del voto de la clase media fue definido no por el miedo económico sino por el antifujimorismo (Levitsky, 2001). En particular, los peruanos que declaran creer en la importancia de garantizar el respeto de los derechos humanos votaron mayoritariamente por Humala sobre Fujimori (Muñoz, 2011).

La contingencia, no obstante, parece ser un factor relacionado más con la oferta política que con la demanda. La elección presidencial mostró que también hay importantes continuidades en las preferencias electorales. En particular, Humala repitió su voto del 2006 con apoyo consistente en zonas rurales y del sur del país (aunque con mejores resultados en zonas urbanas que dicho año). En la primera vuelta, la derecha, representada en el candidato Kuczynski, alcanzó una votación importante en zonas urbanas de la costa donde el 2006 ganó Lourdes Flores Nano. No obstante, mostró aún mayores dificultades de crecer como opción viable fuera de Lima. Sin el APRA en competencia, el voto en el norte fue mucho más disputado, dividiéndose al menos entre tres fuerzas políticas, con Keiko Fujimori a la cabeza en el agregado. En general, Fujimori obtuvo un apoyo considerable en zonas pobres urbanas y en el centro del país. ${ }^{6}$ En la segunda vuelta, la división territorial del voto fue mucho más evidente (Figura 1). Mientras que las provincias costeñas y de la selva central apoyaron mayoritariamente a Fujimori, Humala recibió un voto consistente en el resto del país, particularmente en el sur.

Paradójicamente, entonces, dentro lo fluida que es la política peruana, se observan importantes continuidades en los patrones geográficos del voto. Como se detectara en el 2006 (Muñoz, 2009), el electorado peruano del 2011 está dividido regional y socioeconómicamente (Tanaka, 2011). La geografía del voto expresa así continuidades vinculadas a los efectos regionales desiguales del crecimiento económico bajo políticas neoliberales. Sin embargo, como ha sido señalado por Muñoz (2011) no se trata del surgimiento de una división convencional de clases. A partir del análisis de opinión pública, se constata que se trata de divisiones regionales y socioculturales que no se pueden reducir a meras posiciones socioeconómicas, ideológicas y de nivel educativo. Parte importante del apoyo de Humala es un voto más indígena, masculino y del centrosur del país. Es el voto de disconformidad de un sector de la población del interior del país cansado no sólo de los limitados beneficios que reciben de la economía sino también del maltrato de un sistema político limeño-céntrico (Muñoz, 2011: 12 y 16). El gobierno de García, con poca voluntad y un Estado ineficiente, no hizo mucho por atender directamente las demandas de estos sectores menos "enganchados" al mercado y que demandan una mayor presencia estatal (Vergara, 2007), explicando esta continuidad de elección a elección. No es casual, por tanto, que los dos candidatos que pasaron a la segunda vuelta fueran precisamente aquellos que prometieron acercar más el Estado a dichos sectores (Levitsky, 2011: 89).

6 En el siguiente vínculo se puede acceder a mapas con los resultados de la primera vuelta a diferentes niveles de agregación: http://www.deigualaigual.net/es/actualidad/peru/5128-nuevo-mapa-politico-del-peruresultados-presidenciales-2011 (Consulta 7-3-2011) 
Figura 1: Ganador a nivel provincial en la segunda vuelta

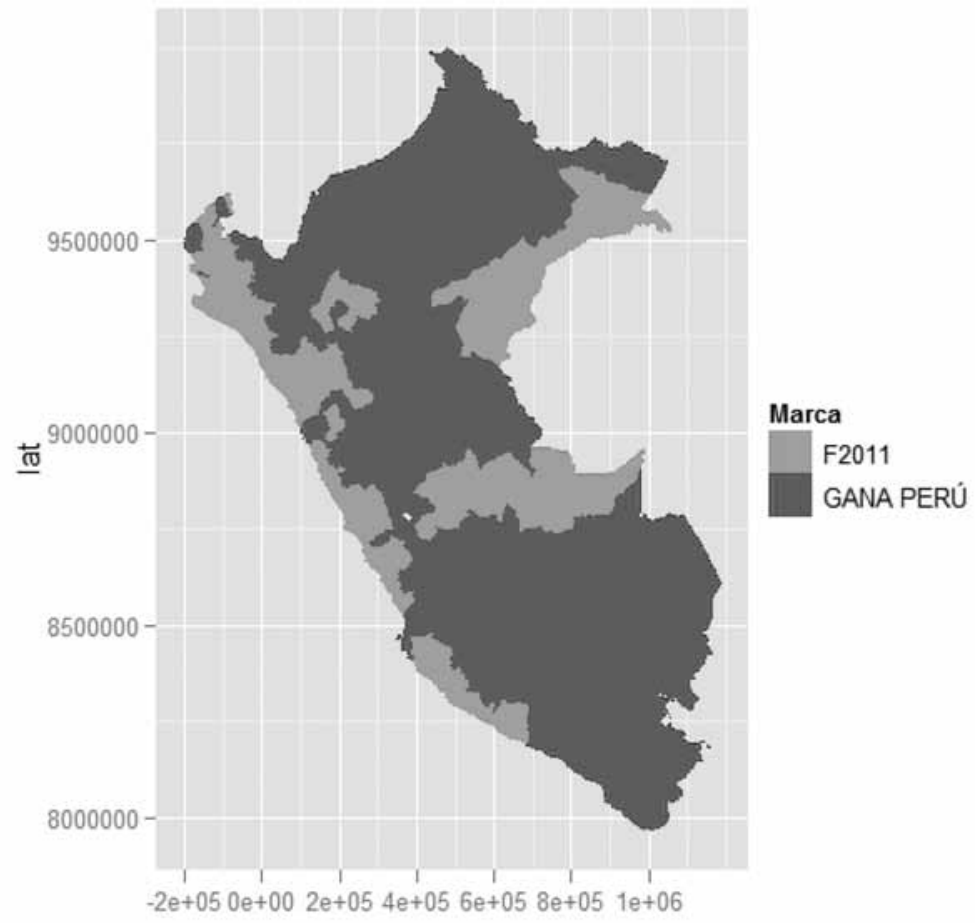

long

\section{PODER EJECUTIVO}

a) Composición y rotación del Gabinete ${ }^{7}$. El 2011 se inicia con un gabinete presidido por el ex ministro de Educación José Chang, que se dedicó básicamente a administrar el gobierno en forma discreta, y preparar las elecciones generales. El último gabinete de Alan García, presidido por la ex ministra de Justicia Rosario Fernández, fue también discreto.

El primer gabinete de Ollanta Humala despertó mucha expectativa pues su composición de alguna manera señalaría la posición ideológica del nuevo gobierno. El gobierno incluyó en el gabinete a diversos grupos que lo apoyaron en la elección, además de técnicos e independientes. El Primer Ministro de Humala fue un empresario cercano a la izquierda y colaborador del Presidente desde el 2006, Salomón Lerner Ghitis. El gabinete fue bastante plural, aunque apenas incluyó un ministro cercano al partido oficialista. 
Como mencionamos antes, Humala tuvo su primera crisis ministerial apenas cuatro meses después de llegar al gobierno. El gabinete presidido por Óscar Valdez mantiene nueve ministros del gabinete Lerner y una composición similar en cuanto a los sectores representados, aunque con menor presencia de la izquierda e incluye dos ministros de la bancada parlamentaria oficialista. En general, sus integrantes tienen un nivel más discreto que los ministros salientes.

b) Análisis del Funcionamiento del Gabinete. El primer gabinete de Humala tenía como una de sus fortalezas su composición plural. Incluía a varias personas de reconocido prestigio e independencia. Pero la pluralidad y autonomía de sus miembros habría conducido a una serie de tensiones a su interior que fueron minando la paciencia del Presidente. Pareciera que el pragmatismo de Humala no va de la mano con las tensiones propias de la pluralidad. Estas tensiones se incrementaron durante la crisis en Cajamarca. Al parecer cansado de estas tensiones, y optando por una postura más unitaria frente al conflicto, Humala toma decisiones contrarias a la opinión de Lerner, motivando su renuncia.

El gabinete Valdez se muestra más unificado que el de su predecesor, aunque también es cierto que hasta el momento no ha tenido retos serios. Valdez es criticado por su aparente incapacidad para dialogar y su falta de reflejos políticos en un contexto en el que los conflictos medioambientales amenazan con reiniciarse.

c) Estilos presidenciales. Humala ha adoptado un estilo pragmático y discreto, distinto al estilo más confrontacional que caracterizó al presidente García. Luego de un discurso inaugural en el que Humala lanzó algunas frases dirigidas al parecer a sus votantes de la primera vuelta (por ejemplo, juró por los valores de la Constitución de 1979, haciendo pensar a sus críticos que intentaría reformar la Constitución de 1993 como señaló en el 2006), ya en la presidencia adoptó una retórica recatada y prudente.

Resalta también la influencia que ejerce sobre Humala su esposa, Nadine Heredia. La Primera Dama ha iniciado una activa campaña de apoyo social, que incluye viajes frecuentes al interior del país. Asimismo, acompaña al Presidente en todas sus actividades oficiales, incluyendo aquéllas en las que las normas protocolares no lo contemplan, como las reuniones con otros Jefes de Estado. Si bien la Ley Electoral prohíbe que los familiares del Presidente puedan ser candidatos, se especula sobre la posibilidad de que se modifique la misma para que Heredia sea candidata en el 2016.

Otro aspecto relevante sobre el estilo presidencial es la presencia de militares en retiro en el entorno de Humala que lo acompañan desde antes de la campaña. Esta cercanía a sectores militares, especialmente a un ex coronel que lo asesora en temas de seguridad, ha dado lugar a quejas y especulaciones por supuesta intervención política en los ascensos y retiros militares y policiales.

Finalmente, un caso que afectó al Poder Ejecutivo fue el escándalo de corrupción que involucró al Segundo Vicepresidente (y congresista) Omar Chehade en octubre, apenas tres meses después de asumir el cargo. El caso, una acusación por un supuesto tráfico de influencias, dio lugar a la renuncia de Chehade a la Vicepresidencia de la República y su suspensión del Congreso. 


\section{PODER LEGISLATIVO}

Como se ha señalado en anteriores reportes, el Congreso peruano tiene serios problemas de legitimidad, transfuguismo y fragmentación. El Congreso es, junto al Poder Judicial, una de las instituciones más desaprobadas por la población. En parte por ese desprestigio, la tasa de reelección del actual Congreso fue de apenas $22 \%$, ligeramente superior a la del anterior Congreso (18\%).

Solo seis agrupaciones o alianzas políticas de las once en carrera pasaron la valla electoral de $5 \%$ y lograron llegar al Congreso (Cuadro 6). Estos grupos finalmente constituyeron seis bancadas (Cuadro 7). Sin embargo, este número moderado de grupos de parlamentarios no garantiza la estabilidad de los mismos durante los próximos cinco años. La debilidad de los partidos políticos hace al Congreso muy fluido, con parlamentarios sin una clara

Cuadro 6: Número de Escaños por Partido

\begin{tabular}{lcc}
\hline Organización política & Escaños & $\%$ \\
\hline Partido Gana Perú & 47 & 36,2 \\
Fuerza 2011 & 37 & 28,5 \\
Partido Perú Posible & 2 & 16,2 \\
Alianza por el Gran Cambio & 12 & 9,2 \\
Alianza Solidaridad Nacional & 9 & 6,9 \\
Partido Aprista Peruano & 4 & 3,1 \\
\hline Total & 130 & 100,0 \\
\hline
\end{tabular}

Fuente: Reflexión Democrática.

Cuadro 7: Grupos Parlamentarios

\begin{tabular}{lcl}
\hline Grupos Parlamentarios & $\begin{array}{c}\text { Número de } \\
\text { Congresistas }\end{array}$ & \multicolumn{1}{c}{ Partidos } \\
\hline Alianza Parlamentaria & 20 & Perú Posible, Acción Popular y Somos Perú \\
Alianza por el Gran Cambio & 12 & $\begin{array}{l}\text { Partido Popular Cristiano, Partido Humanista, } \\
\text { Alianza por el Progreso y Restauración Nacional }\end{array}$ \\
Concertación Parlamentaria & 6 & $\begin{array}{l}\text { Partido Aprista Peruano y dos congresistas que } \\
\text { renunciaron a su bancada }\end{array}$ \\
Fujimorista & 37 & Fuerza 2011 \\
Nacionalista - Gana Perú & 47 & Gana Perú* \\
Solidaridad Nacional & 8 & Alianza Solidaridad Nacional \\
\hline Total Congresistas & 130 & \\
\hline
\end{tabular}

Fuente: Congreso de la República.

* 3 congresistas suspendidos: Anicama, Chehade y Romero. 
fidelidad a los grupos por los que resultaron elegidos. Además, las alianzas electorales permiten que un grupo político incluya a varios partidos en una lista al Congreso, por lo que esta inicial concentración esconde ya más partidos en las bancadas.

El Congreso del 2006, por ejemplo, se inició con cinco grupos y se fue fragmentando mientras avanzaba el lustro, terminando con nueve grupos. El mismo patrón de fragmentación y transfuguismo se observó en el Congreso del 2001 (Tanaka y Barrenechea, 2011). Si bien es muy probable que esta fluidez continúe, creemos que en este período habrá un poco más de estabilidad por la mayor cohesión de las dos bancadas más numerosas. Tanto la bancada de Gana Perú, conformada por el Partido Nacionalista y aliados, como el Fujimorista deberían mantener un mayor control sobre sus miembros por la expectativa de contar con candidatos competitivos en el 2016 (aunque la bancada oficialista muestra ya ciertas resquebrajaduras por la existencia a su interior de un grupo de izquierda más autónomo). En los demás grupos posiblemente sí veamos que se repite este patrón de fragmentación, pues fueron desde el inicio alianzas electorales o listas conformadas con invitados.

Como en años anteriores, la Mesa Directiva del Congreso, compuesta por un presidente y tres vicepresidentes, fue ganada por el oficialismo en alianza con otras bancadas parlamentarias. La primera mesa directiva está compuesta por una alianza multipartidaria liderada por Gana Perú, junto a Alianza Parlamentaria, Alianza por el Gran Cambio y Solidaridad Nacional. En una tendencia que se repite (salvo una excepción en el período 2004-2005), el partido de gobierno sin mayoría es capaz de negociar los votos necesarios para presidir la mesa directiva.

Esta fragmentación y débil cohesión de los grupos en el Congreso también permite al Ejecutivo avanzar sus agendas mediante acuerdos políticos o negociaciones particulares. Desde la transición del año 2000 Ejecutivos sin mayoría en el Congreso no tienen mayor dificultad en avanzar sus intereses. Si bien esta primacía del Ejecutivo es clara, sería exagerado considerar al Congreso como totalmente subordinado, o como incapaz de fiscalizarlo. En determinados contextos los grupos parlamentarios de oposición logran impulsar algunas de sus demandas o explotar los escándalos del gobierno a su favor. Sin embargo, la imagen es la de un Congreso reactivo, marcado por la agenda del Ejecutivo.

Un factor que explica la baja aprobación del Congreso son los continuos escándalos de sus miembros, los cuales dan lugar a pedidos de levantamiento de la inmunidad parlamentaria o procesos internos disciplinarios. A pesar del poco tiempo en funciones este Congreso ya cuenta con tres congresistas suspendidos. Estas constantes denuncias se explican en parte por la forma en que se conforman las listas del Congreso: partidos sin recursos y sin candidatos competitivos llegan a acuerdos con políticos independientes más competitivos electoralmente y/o con recursos. Casi no existen filtros sobre estos candidatos, pues los partidos no los realizan y los organismos electorales no fiscalizan en detalle sus antecedentes. Sin partidos sólidos parece imposible escapar de este círculo vicioso: la debilidad partidaria conduce a bancadas frágiles, transfuguismo y malos representantes, que aumentan el desprestigio del Congreso y a su vez de los partidos políticos. 


\section{RELACIÓN ENTRE LOS DISTINTOS NIVELES DE GOBIERNO}

En el Perú las autoridades de niveles subnacionales tienen poco poder como actores nacionales, incluidos los presidentes regionales. La regionalización del 2002 no ha dado lugar a centros de poder en las regiones que disputen la primacía política de Lima (Vergara, 2012). Una medida indirecta de ello es, por ejemplo, el papel secundario de los presidentes regionales en las elecciones presidenciales.

En realidad, salvo excepciones, los presidentes regionales no suelen tener mucho poder dentro de sus regiones. Esto es también llamativo desde una perspectiva comparada. La municipalización ha sido el tipo de descentralización más profundizado y sostenido en el Perú desde la transición democrática de 1980. Dado el diseño institucional municipal vigente, Perú cuenta con dos niveles de gobierno local (provincial y distrital) completamente autónomos entre sí y también del gobierno regional. Gobiernos regionales, municipalidades provinciales y municipalidades distritales reciben sus transferencias directamente del gobierno central. Esto, sumado a la falta de una clara jerarquía de competencias entre niveles de gobierno, hace que en la práctica a menudo los presidentes regionales terminen compitiendo por poder con los alcaldes provinciales, particularmente el alcalde de la provincia capital de departamento. A su vez, el Ejecutivo nacional suele aprovechar estos fueros independientes de poder y establecer negociaciones individuales con las autoridades subnacionales para las decisiones de inversión del gobierno central. Esto debilita aún más a los presidentes regionales.

Ni siquiera el crecimiento económico de algunas regiones permite hablar de poder regional significativo en la periferia. Esta situación se explica en parte por la alta centralización en el país: las grandes decisiones de inversión se toman en Lima. Pero también, creemos, porque no se observan élites económicas con intereses contrapuestos a los del centro en la periferia, ni élites locales con poder económico comparable a las del centro. Por ahora ni los políticos ni las élites económicas regionales son actores políticos muy relevantes.

Sin embargo, resaltar la primacía del gobierno central no implica que el Ejecutivo pueda imponer su agenda a nivel local, pues el Estado central también tiene problemas serios de debilidad institucional. En general, en un contexto de debilidad partidaria, política personalista y alta competencia política, la coordinación entre niveles de gobierno se torna sumamente compleja y conflictiva.

La mayor tensión entre el gobierno nacional y un gobierno subnacional en el 2011 se dio en el caso del Conflicto de Conga. Durante dicho conflicto el Ejecutivo usó por primera vez un arma de presión financiera: congeló por unos días los fondos del gobierno regional y solicitó al Ministerio Público investigar si estos fondos se habían usado para promover las protestas. La ley no incluye medidas de este tipo, aunque el Ejecutivo interpretó que sí estaba en la potestad de hacerlo al ser parte de sus funciones de transferencia presupuestal. En una reunión convocada a los pocos días, los presidentes regionales actuaron preventivamente para evitar que dicho mecanismo pueda utilizarse en el futuro y manifestaron su apoyo al presidente regional de Cajamarca, calificando la decisión como un exceso. 


\section{EVALUACIÓN GENERAL SOBRE EL FUNCIONAMIENTO Y CALIDAD DE LA DEMOCRACIA}

Desde la caída del fujimorismo el país cumple con los requisitos para ser considerado una democracia. Sin embargo, creemos que la calidad de dicha democracia todavía deja mucho que desear. El problema más mencionado durante los últimos años sobre la calidad de la democracia peruana tiene que ver con la divergencia entre el programa del gobierno elegido y su forma de gobernar, generalmente más a la derecha que los electores. Las preferencias electorales no determinarían claramente el contenido del programa de gobierno.

Coincidimos en parte con esta apreciación. Alejandro Toledo gobernó un poco más a la derecha de lo prometido en la campaña del 2001. Alan García gobernó bastante más a la derecha de lo prometido en su campaña del "cambio responsable" del 2011. Y todo indica que Ollanta Humala mantendrá un rumbo similar, gobernando incluso más a la derecha de su posición moderada de la segunda vuelta de la elección. Se señala en broma que la mayoría de ministros que hoy tiene Humala no votaron por él en segunda vuelta. ¿Qué explica este movimiento hacia la derecha una vez que los presidentes empiezan a gobernar?

Creemos que esta derechización de los gobiernos en materia económica se debe, en primer término, a un electorado fragmentado. Simplificando las cosas, podemos decir que en las últimas tres elecciones un $20 \%$ vota más hacia la derecha y en forma conservadora, un $35 \%$ ha votado por un cambio más substantivo. Pero el $45 \%$ restante parece apoyar cambios moderados o es renuente a un cambio drástico en términos económicos. Hay una base social que limita agendas de izquierda muy radicales. La agenda con la que Humala inició la elección no era la de la mayoría de votantes que votó por la derecha o por opciones más centristas en materia económica. Sin embargo, esta base social no explica por qué tres presidentes hayan terminado bastante más a la derecha que su posición en la campaña: este "centro" económico permitiría posiciones más reformistas.

Lo que consideramos termina de "jalar" a los gobernantes hacia ese lado del espectro político es la mayor organización, influencia y recursos del empresariado beneficiado por el actual modelo económico y la existencia de una tecnocracia estable en los centros de decisión económica. No creemos que la conducta del Ejecutivo se explique tan solo por una "captura" del Estado, a pesar del poder de los grupos económicos y sonados casos de influencia corrupta en el gobierno. Creemos que es más bien la mayor organización y recursos de estos grupos para influenciar políticamente al gobierno y la debilidad relativa de otros grupos que se oponen a sus intereses. En efecto, al otro lado del espectro, representando a los más críticos de la economía y el Estado, no hay ni partidos políticos ni organizaciones con poder o recursos para mantener una influencia constante en la política. Ni siquiera el "centro" (tomando este término en forma amplia) está representado por partidos organizados.

En esas condiciones, afectado por protestas locales y la necesidad de mantener la economía en crecimiento para mantener estable al gobierno, los presidentes parecen 
buscar seguridad en el lado derecho organizado. Incluso un gobierno como el actual que ganó contra estos intereses económicos y que ha tomado algunas medidas contrarias a los mismos, termina acercándose a estas preferencias. El resultado es la continuidad, para bien o para mal, del mismo tipo de política económica en el Perú desde los años de Alberto Fujimori. ${ }^{8}$ Como señalamos más abajo, esta "derechización" no necesariamente implica que Humala se esté apartando de toda su base electoral de primera vuelta, pues podría estar respondiendo por medio de programas sociales a las demandas de un sector importante de dichos votantes.

La conducta de los medios de comunicación durante la elección del 2011 mostró a estos medios demasiado cerca a los intereses empresariales, limitando una competencia electoral justa. La mayoría de los medios de comunicación escritos y televisivos apostaron abiertamente contra la candidatura de Ollanta Humala por su posición crítica frente al modelo económico. No hubo fiscalización similar con las otras candidaturas, incluso aquellas que dejaba similares o mayores dudas democráticas (García Llorens, 2011).

A pesar de estos patrones negativos, hay algunos aspectos que consideramos positivos para la democracia en estos primeros meses de gobierno. A diferencia de sus antecesores, Humala inició el gobierno respondiendo directamente a sus promesas de campaña. Para comenzar, elevó el salario mínimo y, como vimos, se aprobó el gravamen minero y la Ley de Consulta Previa. Éstas eran medidas que sus críticos resaltaron como muy difíciles de cumplir.

Del mismo modo, el actual gobierno tiene una agenda más ambiciosa para mejorar la igualdad de oportunidades. Tal vez sean estas medidas las que le hayan permitido mantener su popularidad alta entre sectores populares hasta el momento. Conforme a sus promesas, el gobierno ha iniciado programas sociales para mejorar la igualdad de oportunidades en el país. Desde el Ministerio de Desarrollo e Inclusión Social se han impulsado los programas Pensión 65, el cual en la actualidad atiende a cerca de 40.000 personas mayores de 65 años. Asimismo, se incrementó la cobertura del programa de transferencias condicionadas JUNTOS en los departamentos de extrema pobreza. Se busca ampliar los beneficiarios de 500.000 hogares en la actualidad a 746.000 en el 2016. Del mismo modo, se ha creado e implementado un programa de apoyo a niños de 0 a 3 años (Cuna Más) que busca atender a 250.000 niños en el 2016. Por otro lado, el programa Beca 18 impulsado por el Ministerio de Educación tiene por objetivo apoyar a unos 5.000 jóvenes con becas integrales para realizar estudios superiores en el 2012, y llegar a 25.000 en el 2016. ${ }^{9}$ Además de estos programas, otro factor que puede ayudar a la popularidad, aunque parezca anecdótico, son los frecuentes viajes fuera de Lima tanto de Humala como de Nadine Heredia, un estilo que contrasta con la menor presencia fuera de Lima de García y Toledo.

8 Una explicación más elaborada de esta continuidad, que resalta estos factores sociales y organizativos, además de otros, la presentan Vergara y Encinas 2012.

9 Exposición del Primer Ministro Óscar Valdez al Congreso de la República, 5 de enero del 2012. http:// snipnet.mef.gob.pe/contenidos/politicas/lineamientos/1_nacional/Exposici_de_la_Poltica_General_del_ Gobiernoyprincipalesmedidas_de_ges 
Más allá de las dudas que dejan sus asesores militares y algunos gestos de mano dura, hasta el momento Humala no ha dado signos de ser un riesgo para la continuidad democrática o económica como se especulaba dados sus antecedentes del 2006. La pregunta de si será Chávez o Lula parece haber quedado en el olvido, y hoy se discute más bien si terminará como Toledo o García, con una aprobación mayoritaria urbana y cercano a la derecha, o si logrará construir una posición de "centro" con llegada a diversos sectores sociales y regionales que legitime a una democracia muy alicaída entre la población, especialmente entre los sectores más críticos.

\section{REFERENCIAS}

Banco Mundial. 2008. What do the 2004-2007 poverty trends in Peru tell us? Lessons for the emerging policy and research agenda. Washington, DC: The International Bank for Reconstruction and Development / World Bank.

Cooperacción. 2010. "Informe Cartográfico Sobre Concesiones Mineras en el Perú". (En Línea) http:/ / www.redge.org.pe/sites/default/files/js/5.\%20Presentacioninforme.pdf (Consulta 1-3-2012).

Cyr, Jennifer. 2011. “¿Por qué el APRA no Muere?”. En Post-Candidatos: Guía Analítica de Supervivencia hasta las Próximas Elecciones. Compilado por Carlos Meléndez. Lima: Mitin y 50+1, 197-226.

De Gramont, Diane. 2010. Leaving Lima behind? The victory and Evolution of Regional Parties in Peru, Tesis de Bachillerato. Cambridge: Harvard University.

García Llorens, Mariel. 2011. “El Gran Show Electoral. Las Claves Espectaculares de la Política Mediatizada". En Post-Candidatos: Guía Analítica de Supervivencia hasta las Próximas Elecciones. Compilado por Carlos Meléndez. Lima: Mitin y 50+1, 285-336.

León, Carlos. 2011. "Nosotros nos Equivocamos Menos. Vida, Muerte y Resurrección Electoral de Ollanta Humala". En Post-Candidatos: Guía Analítica de Supervivencia hasta las Próximas Elecciones. Compilado por Carlos Meléndez. Lima: Mitin y 50+1, 43-90.

Levitsky, Steven. 2011. "A Surprising Left Turn”. Journal of Democracy 22 (4): 84-94.

Levitsky, Steven and Cameron Maxwell. 2003. "Democracy Without Parties? Political Parties and Regime Change in Fujimori's Peru". Latin American Politics and Society, 45 (3):1-33.

Meléndez, Carlos. 2011. "Perder es Cuestión de Método. Lecciones del Fracaso Electoral de Luis Castañeda". En Post-Candidatos: Guía Analítica de Supervivencia hasta las Próximas Elecciones. Compilado por Carlos Meléndez. Lima: Mitin y 50+1, 173-196.

Meléndez, Carlos y Carlos León. 2010. "Perú 2009: Los Legados del Autoritarismo". Revista de Ciencia Politica 30 (2): 451-477.

Meléndez, Carlos y Carlos León. 2009. “Perú 2008: El Juego de Ajedrez de la Gobernabilidad en Partidas Simultáneas". Revista de Ciencia Política 29 (2): 591-609.

Muñoz, Paula. 2011. "Más Allá de la Campaña". Opinión y Análisis. (Elecciones presidenciales segunda vuelta: un análisis del voto peruano. Documento del Instituto de Opinión Pública de la Pontificia Universidad Católica del Perú, Año II (julio): 9-16.

Muñoz, Paula y Andrea García. 2011. “Tendencias, Particularidades y Perfil de los Candidatos Más Exitosos". En Perú Debate: El Nuevo Poder en las Regiones, Lima: PUCP, 8-17.

Navarro, Melissa. 2011. “Tras el líder: Oportunidades de un partido personalista para lograr la continuidad luego del alejamiento del líder fundacional: el caso del fujimorismo". Politai. Revista de Ciencia Política 2 (3): 139-148.

Remy, María Isabel. 2011. “Un Balance Final de las Elecciones Municipales y Provinciales: en qué Punto Quedaron los Partidos Políticos? Revista Argumentos 5 (1). Disponible en: http:/ / www.revistargumentos. org.pe/un_balance_final_de_las_elecciones_municipales_y_regionales_html

Remy, María Isabel. 2011. "Elecciones Regionales 2010 o el Sueño de la Candidatura Propia". Revista Argumentos 4 (3). Disponible en: http://revistargumentos.org.pe/elecciones_regionales_2010_o_ el_sueno_de_la_candidatura_propia.html 
Tanaka, Martín. 2011. "A Vote for Moderate Change". Journal of Democracy 22 (4): 84-94.

Tanaka, Martín. 2005. Democracia sin Partidos: Perú 2001-2005. Lima: Instituto de Estudios Peruanos.

Tanaka, Martín y Yamilé Guibert. 2011. “Entre la Vaporización de los Partidos y la Debilidad de los Movimientos Regionales". En Perú Debate: El Nuevo Poder en las Regiones. Lima: PUCP, 18-29.

Tanaka, Martín y Rodrigo Barrenechea. 2010. "Evaluando la oferta de Partidos: ¿cuál es el perfil de los candidatos al próximo Parlamento?" Revista Argumentos, 5 (1). Disponible en: http://www. revistargumentos.org.peevaluando_la_oferta_de_los_partidos_.html

Tanaka, Martín y Sofía Vera. 2008. "El Neodualismo de la Política Peruana". Revista de Ciencia Política 28 (1): 347-365.

Urrutia, Adriana. 2011. “Que la Fuerza (2011) esté con Keiko: el Nuevo Baile del Fujimorismo. El Fujimorismo, su Organización y sus Estrategias de Campaña". En Post-Candidatos: Guía Analítica de Supervivencia hasta las Próximas Elecciones. Compilado por Carlos Meléndez. Lima: Mitin y 50+1,91-120.

Vera, Sofía. 2011. "Volar sin Paracaídas: Alejandro Toledo y su Reelección Frustrada". En Post-Candidatos: Guía Analítica de Supervivencia hasta las Próximas Elecciones. Compilado por Carlos Meléndez. Lima: Mitin y 50+1, 147-172.

Vera, Sofía. 2010. "Radiografía a la política en las regiones: tendencias a partir de la evidencia de tres procesos electorales (2002, 2006 y 2010)". Revista Argumentos 4 (5). Noviembre. Disponible en: http:/ / www.revistargumentos.org.pe/index.php?fp_verpub=true\&idpub=395

Vergara, Alberto y Daniel Encinas. 2012. "La sorpresiva continuidad. Esbozo de los mecanismos de reproducción de la herencia institucional del fujimorato". Ponencia presentada en el Seminario "Ecuador, Bolivia y Perú. Nuevos y viejos escenarios, una perspectiva comparada". Quito, 14-16 de marzo.

Vergara, Alberto. 2011. “United by Discord, Divided by Consensus: National and Sub-National Articulation in Bolivia and Peru, 2000-2010". Journal of Politics in Latin America 3 (3): 65-93.

Vergara, Alberto. 2011. “¿Qué es el APRA Hoy?”. En Anticandidatos. Compilado por Carlos Meléndez. Lima: Mitin y 50+1, 41-60.

Vergara, Alberto. 2007. “Ni Amnésicos Ni Irracionales: la Elección del 2006". En Perspectiva Histórica. Lima: Solar. 
Anexo

\begin{tabular}{|c|c|c|c|c|}
\hline \multirow{2}{*}{ Ministerio } & \multicolumn{2}{|c|}{ Gabinete Lerner (28/07/11 a 11/12/11) } & \multicolumn{2}{|c|}{ Gabinete Valdez (11/12/11) } \\
\hline & Nombre & Partido & Nombre & Partido \\
\hline PCM & Salomón Lerner Ghitis & Gana Perú & Óscar Valdez Dancuart & Ind. \\
\hline Economía & Luis Miguel Castilla & Ind. & Ratificado & \\
\hline Vivienda & René Cornejo & Ind. & Ratificado & \\
\hline $\begin{array}{l}\text { Desarrollo e Inclusión } \\
\text { Social }\end{array}$ & Carolina Trivelli* & Ind. & Ratificado & \\
\hline Salud & Alberto Tejada & Ind. & Ratificado & \\
\hline $\begin{array}{l}\text { Transportes y } \\
\text { Comunicaciones }\end{array}$ & Carlos Paredes & Ind. & Ratificado & \\
\hline $\begin{array}{l}\text { Comercio Exterior y } \\
\text { Turismo }\end{array}$ & Luis Silva & Ind. & Ratificado & \\
\hline Relaciones Exteriores & Rafael Roncagliolo & Ind. & Ratificado & \\
\hline Educación & Patricia Salas & Ind. & Ratificado & \\
\hline Agricultura & Miguel Caillaux & Ind. & Luis Ginocchio & Ind. \\
\hline Cultura & Susana Baca & Ind. & Luis Peirano & Ind. \\
\hline Ambiente & Ricardo Gisecke & Ind. & Manuel Pulgar-Vidal & Ind. \\
\hline Energía y Minas & Carlos Herrera Descalzi & Ind. & Jorge Merino & Ind. \\
\hline Mujer & Aída García Naranajo & Partido Socialista & Ana Jara & Gana Perú \\
\hline Justicia & Francisco Eguiguren & Ind. & Juan Jiménez & Ind. \\
\hline Interior & Óscar Valdez Dancuart & Ind. & Daniel Lozada & Ind. \\
\hline Producción & Kurt Burneo & Ind. & José Urquizo & Gana Perú \\
\hline Trabajo & Rudecindo Vega & Perú Posible & José Villena & Ind. \\
\hline Defensa & Daniel Mora & Perú Posible & Alberto Otárola & Ind. \\
\hline
\end{tabular}

* Nuevo ministerio. Nombrada el 21/10/11.

Eduardo Dargent. Pontificia Universidad Católica del Perú.

E-mail: edargent@pucp.edu.pe

Paula Muñoz. Universidad de Texas en Austin.

E-mail: pmunoz@utexas.edu 